\title{
How Profitable are Strategic Behaviors in a Market?
}

\author{
Ning Chen ${ }^{1}$, Xiaotie Deng ${ }^{2}$, Jie Zhang ${ }^{3}$ \\ 1 Division of Mathematical Sciences, School of Physical and Mathematical Sciences, \\ Nanyang Technological University, Singapore. \\ ningc@ntu.edu.sg \\ 2 Department of Computer Science, University of Liverpool, UK. \\ xiaotie@liv.ac.uk \\ 3 Department of Computer Science, City University of Hong Kong, Hong Kong. \\ csjiezhang@gmail.com
}

\begin{abstract}
It is common wisdom that individuals behave strategically in economic environments. We consider Fisher markets with Leontief utilities and study strategic behaviors of individual buyers in market equilibria. While simple examples illustrate that buyers do get larger utilities when behaving strategically, we show that the benefits can be quite limited: We introduce the concept of incentive ratio to capture the extent to which utility can be increased by strategic behaviors of an individual, and show that the incentive ratio of Leontief markets is less than 2. We also reveal that the incentive ratios are insensitive to market sizes. Potentially, the concept incentive ratio can have applications in other strategic settings as well.
\end{abstract}

\section{Introduction}

Market equilibrium is a vital notion in classical economic theory. Understanding its properties and computation has been one of the central questions in Algorithmic Game Theory. For the Fisher market model [7], we consider pricing and allocation of $m$ products to $n$ buyers, each with an initially endowed amount of cash $e_{i}$ and with a non-decreasing concave utility function. At a market equilibrium, all products are sold out, all cash is spent, and, most importantly, the set of products purchased by each buyer maximizes his utility function for the given equilibrium price vector constrained by his initial endowment. It is well-known that a market equilibrium always exists given mild assumptions on the utility functions $[5,7]$.

A critical assumption in studying and analyzing marketplaces is that all individuals are rational and would like to seek their utility maximized. In a market equilibrium, while it is true that every buyer gets his maximum utility through an equilibrium allocation, his utility function indeed takes an effect on generating equilibrium prices, which in turn affect his utility in the equilibrium allocation. In other words, (strategic) buyers may affect market equilibria and their own utilities through the information that the market collects. 
From a different angle, market equilibrium maps input information from buyers to prices and allocations; this defines a natural market equilibrium mechanis$m$, in which buyers report to the market their utility functions (their strategies consist of all possible functions in a given domain) and the market generates an equilibrium output for the reported functions. Adsul et al. [1] recently observed that bidding truthfully is not a dominant strategy in the market equilibrium mechanism for linear utility functions. That is, buyers can behave strategically in a linear market to increase their utilities. This phenomenon is not restricted to the linear utility functions. The following example suggests that it may happen for other utility functions as well, e.g., Leontief functions $[9,10,16]$ - the utility to buyer $i$ is given by $u_{i}=\min _{j}\left\{\frac{x_{i j}}{a_{i j}}\right\}$, where $x_{i j}$ is the allocation of item $j$ to $i$ and $a_{i j}$ 's are parameters associated with buyer $i$.

Example 1. There are two buyers $i_{1}$ and $i_{2}$ with endowment $e_{1}=2 / 3$ and $e_{2}=$ $1 / 3$ respectively. There are two items $j_{1}$ and $j_{2}$ both with unit quantity. Buyer $i_{1}$ 's utility is $u_{1}=\min \left\{\frac{x_{11}}{\frac{2}{3}}, \frac{x_{12}}{\frac{1}{3}}\right\}$, and buyer $i_{2}$ 's utility is $u_{2}=\min \left\{\frac{x_{21}}{\frac{1}{3}}, \frac{x_{22}}{\frac{2}{3}}\right\}$. Then in an equilibrium output ${ }^{4}$ with price vector $\mathbf{p}=(1,0)$ and allocations $\mathbf{x}_{1}=\left(\frac{2}{3}, \frac{1}{3}\right)$ and $\mathbf{x}_{2}=\left(\frac{1}{3}, \frac{2}{3}\right)$, the utilities are $u_{1}=1$ and $u_{2}=1$. Now if buyer $i_{1}$ strategizes in the market and reports $u_{1}^{\prime}=\min \left\{\frac{x_{11}}{\frac{5}{9}}, \frac{x_{12}}{\frac{4}{9}}\right\}$, price vector $\mathbf{p}^{\prime}=(0,1)$ and allocations $\mathbf{x}_{1}^{\prime}=\left(\frac{5}{6}, \frac{2}{3}\right)$ and $\mathbf{x}_{2}^{\prime}=\left(\frac{1}{6}, \frac{1}{3}\right)$ give an equilibrium for the new setting. Now the utilities are $u_{1}^{\prime}=\frac{5}{4}$ and $u_{2}^{\prime}=\frac{1}{2}$, where buyer $i_{1}$ gets a strictly larger utility.

These intriguing phenomena motivate us to consider the following questions: How much can utility be increased with strategic behaviors in a market? How does the answer to the question depend on the domains of utility functions? The answers to these questions would help us to understand the effects of strategic behaviors in marketplaces on their equilibria and utilities of individuals. In this paper, we take a first step towards answering these questions by considering Leontief utility functions. Leontief utilities are a special case of well-studied Constant Elasticity of Substitution (CES) functions [4] and represent perfect complementarity preferences of buyers. They allow us to model a wide range of realistic preferences of buyers, illustrating a variety of different phenomena of markets.

We study this problem by introducing a quantity called incentive ratio, which characterizes the extent to which utilities can be increased by strategic behaviors of individuals. Formally, for any fixed bids of other buyers in a given market, the incentive ratio of any given buyer is defined to be his maximum possible utility by behaving strategically divided by his utility when bidding truthfully, given any fixed bids of all other buyers. Note that the definition of incentive ratio can be generalized to any mechanisms. Indeed, if a mechanism is incentive

\footnotetext{
${ }^{4}$ In a Leontief market, equilibrium allocation may not be unique, but the utility of every buyer in all equilibria, evaluated using the utility functions reported, is unique [24]. Therefore, the selection of output equilibria will not affect the utility of any buyer.
} 
compatible, its incentive ratio is one. A mechanism/market with a small incentive ratio implies that the "invisible hand" in the market is strong in the sense that no one can benefit much from (complicated) strategic considerations, even if one has complete information of the others. Incentive ratio therefore characterizes robustness of incentives in a mechanism/market, and has potential applications in other incentive scenarios.

A related concept, price of anarchy [19], together with several variants (e.g., price of stability [2]), is becoming one of the most important solution concepts in our understanding of Algorithmic Game Theory. While the price of anarchy considers the ratio of the social welfare achieved in the worst case Nash equilibrium versus the optimum social welfare, our concept of incentive ratio does not deal with social welfare but that of individual optimality, for everyone. It is close to the approximation market equilibrium price introduced in [11] where every individual achieves a solution within a constant factor from its own optimum under the given price. In both concepts, individuals do not achieve their own optimum but bounded by a constant factor away. However, in [11], the consideration is about computational difficulty, but, in our model, we consider an exact equilibrium mechanism when market participants may play strategically.

In this paper, we show that the incentive ratio of Leontief markets is less than 2. Our proof lies on revealing interconnections of the incentive ratios of markets with different sizes. In particular, we prove that the incentive ratio is independent of the number of buyers (Theorem 5), by showing a reduction from any $n$-buyer market to a 2-buyer market, and vice versa. A similar result holds for items as well, i.e., the incentive ratio is independent of the number of items (Theorem 6). These results are of independent interests and imply that the incentive ratio is insensitive to the size of a market. In other words, the size of a market is not a factor to affect the largest possible utility that a strategic individual can obtain. Given these properties, we therefore focus on a market with 2 buyers and 2 items to bound the incentive ratio. Our proof involves in best response and dominant strategy analysis for Leontief markets.

\section{$1.1 \quad$ Related work}

Market equilibrium, especially its algorithmic perspective, has received extensive attention in the last decade $[11,17,10,25,12]$. For Fisher markets, Devanur et al. [13] developed a polynomial primal-dual algorithm with linear utility functions. Codenotti and Varadarajan [9] modeled the Leontief market equilibrium problem as a concave maximization problem; Garg et al. [16] gave a polynomial time algorithm to compute an equilibrium for Leontief utilities. Other algorithmic results of computing a market equilibrium in Fisher markets including, e.g., Cobb-Douglas utilities [14] and logarithmic utilities [8].

Roberts and Postlewaite [21] observed that as the number of buyers becomes large, the Walrasian allocation rule is asymptotically incentive compatible. This has a similar flavor to our results that the incentive ratio does not increase as the size of the market enlarges. In a recent paper, Adsul et al. [1] studied strategic behaviors in a Fisher market with linear utilities; they showed that all Nash 
equilibria in their setting are conflict-free, i.e., buyers have no conflict on allocations that maximize their utilities. They also showed that a symmetric strategy profile, i.e., all buyers report the same utility function, is a Nash equilibrium if and only if it is conflict-free. Our paper however has completely different focuses as we study Leontief rather than linear utilities, and we consider strategic behaviors for each individual "locally" whereas [1] studied Nash equilibrium for all the buyers.

\section{Preliminaries}

In a Fisher market $M$, we are given a set of $n$ buyers and a set of $m$ divisible items. Without loss of generality, assume that all items are of unit quantity supply. Each buyer $i$ has an initial endowment $e_{i}>0$, which is normalized to be $\sum_{i} e_{i}=1$, and a utility function $u_{i}\left(\mathbf{x}_{i}\right)$, where $\mathbf{x}_{i}=\left(x_{i 1}, \ldots, x_{i m}\right)$ is a vector denoting the amount of items that $i$ receives.

One of the most important classes of utility functions is that of Constant Elasticity of Substitution (CES) functions [23]: $u_{i}\left(\mathbf{x}_{\mathbf{i}}\right)=\left(\sum_{j=1}^{m} a_{i j} x_{i j}^{\rho}\right)^{\frac{1}{\rho}}$, for $-\infty<\rho<1$ and $\rho \neq 0$, where $\mathbf{a}_{\mathbf{i}}=\left(a_{i 1}, \ldots, a_{i m}\right)$ is a given vector associated with each buyer; its elements $a_{i j}>0$. Let $a_{i}^{\max }=\max _{j}\left\{a_{i j}\right\}$ and $a_{i}^{\min }=\min _{j}\left\{a_{i j}\right\}$. CES functions allow us to model a wide range of realistic preferences of buyers, and have been shown to derive, in the limit, special cases including, e.g., linear, Leontief, and Cobb-Douglas utility functions [4].

The output of a market is given by a tuple $(\mathbf{p}, \mathbf{x})$, where $\mathbf{p}=\left(p_{1}, \ldots, p_{m}\right)$ is a price vector and $\mathbf{x}=\left(\mathbf{x}_{1}, \ldots, \mathbf{x}_{n}\right)$ is an allocation vector. We say $(\mathbf{p}, \mathbf{x})$ is a market equilibrium (and $\mathbf{p}$ equilibrium price and $\mathbf{x}$ equilibrium allocation respectively) if the following conditions hold:

- The allocation $\mathbf{x}_{i}$ maximizes the utility of each buyer $i$ given his endowment $e_{i}$ and price vector $\mathbf{p}$.

- The market is clear, i.e., all items are sold out and all endowments are exhausted. That is, for any item $j, \sum_{i} x_{i j}=1$; and for any buyer $i, \sum_{j} p_{j} x_{i j}=$ $e_{i}$. (Note that this implies that $\sum_{j} p_{j}=\sum_{i} e_{i}=1$.)

For CES functions, the equilibrium allocation can be captured by the seminal Eisenberg-Gale convex program [15].

In this paper, we will focus on Leontief utility functions: The utility of every buyer is given by $u_{i}\left(\mathbf{x}_{\mathbf{i}}\right)=\min _{j}\left\{\frac{x_{i j}}{a_{i j}}\right\}$, where $a_{i j}>0$. Leontief utility function indicates perfect complementarity between different items and is the case when $\rho \rightarrow-\infty$ in the CES functions. Codenotti and Varadarajan [9] gave the following convex program to encode a Leontief market:

$$
\begin{array}{ll}
\max & \sum_{i=1}^{n} e_{i} \log u_{i} \\
\text { s.t. } & \sum_{i=1}^{n} a_{i j} u_{i} \leq 1, \quad \forall j=1, \ldots, m \\
& u_{i} \geq 0, \quad \forall i=1, \ldots, n
\end{array}
$$


Recall that the KKT conditions [6] are necessary for a feasible solution of a nonlinear program to be optimal, provided that some regularity conditions are satisfied. If the objective function and the inequality constraints are continuously differentiable convex functions and the equality constraints are affine functions, the KKT necessary conditions are also sufficient for optimality. Moreover, if the objective function is strictly convex, then the optimal solution is unique [6]. Note that (1) satisfies all the necessary conditions; thus, it possesses all these properties. In particular, this implies that the utility of every buyer is unique in all market equilibria.

Applying the KKT necessary conditions on (1), we get

$$
\left\{\begin{aligned}
-\frac{e_{i}}{u_{i}}+\sum_{j=1}^{m} p_{j} a_{i j}-\mu_{i} & =0, \quad \forall i \\
\mu_{i} u_{i} & =0, \quad \forall i \\
p_{j}\left(\sum_{i=1}^{n} a_{i j} u_{i}-1\right) & =0, \quad \forall j
\end{aligned}\right.
$$

where the Lagrangian variable $p_{j}$ is actually an equilibrium price of the market. Since $u_{i}>0$, we have $\mu_{i}=0$ and $u_{i}=\frac{e_{i}}{\sum_{j=1}^{m} p_{j} a_{i j}}$.

We have the following simple characterization on the uniqueness of allocation in a Leontief market.

Lemma 1. The equilibrium allocation is unique if and only if the first constraint of the convex program (1) is tight. That is, $\sum_{i=1}^{n} a_{i j} u_{i}=1$, for $j=1, \ldots, m$.

\section{Market Equilibrium Mechanism}

Market equilibrium provides a natural mirror that maps input information of a market (i.e., endowments and utility functions) to an output (i.e., prices and allocations). The participation of every buyer in the market is to seek for a utility-maximized allocation. That is, for any given price vector $\mathbf{p}$, every buyer would like to get an allocation that maximizes his utility subject to the budget constraint. This fact is indeed captured by the market equilibrium solution concept.

If we consider rational behaviors of buyers, however, as observed in Example 1 , they may actually behave strategically to increase their utilities. This suggests a natural market equilibrium mechanism, in which buyers report to the market their utility functions ${ }^{5}$ (their strategy space consists of all possible functions in a given domain, i.e., Leontief functions considered in this paper), and then the market generates an equilibrium output based on the reported functions. Formally, each buyer $i$ has a private vector $\mathbf{a}_{\mathbf{i}}=\left(a_{i 1}, \ldots, a_{i m}\right)$,

\footnotetext{
${ }^{5}$ We assume that buyers do not play with their budget and always report the true value $e_{i}$. This assumption is without loss of generality as we are able to show that for any reported utility functions, bidding the true budget is always a dominant strategy.
} 
which denotes his true Leontief utility function, and may bid an arbitrary vector $\mathbf{b}_{\mathbf{i}}=\left(b_{i 1}, \ldots, b_{i m}\right)$ where each $b_{i j}>0$ to the mechanism. Upon receiving the reported vectors $\mathbf{b}_{\mathbf{1}}, \ldots, \mathbf{b}_{\mathbf{n}}$ (as well as endowments) from all buyers, the mechanism outputs prices and allocations of the items; the true utility that buyer $i$ obtains from the output is denoted by $u_{i}\left(\mathbf{b}_{\mathbf{1}}, \ldots, \mathbf{b}_{\mathbf{n}}\right)$.

In this section, we will establish a few properties regarding best response and dominant strategy for the market equilibrium mechanism. Since bidding truthfully is not necessarily a dominant strategy, for any given fixed reported functions of other buyers, every individual buyer will report a function that maximizes his own utility; such a bidding function is called a best response. Before considering best response strategies, we first notice that since equilibrium allocations may not be unique for given reported utility functions, the real utility that a strategic buyer obtains depends on different allocations, which in turn affects his strategic behaviors. The following claims, however, indicate that there is always a best response such that the resulting equilibrium allocation is unique. In the following we denote $b_{i}^{\max }=\max _{j}\left\{b_{i j}\right\}, b_{i}^{\min }=\min _{j}\left\{b_{i j}\right\}, \forall i$.

Theorem 1. For any given market with two buyers $i_{1}, i_{2}$ and any reported bid $\mathbf{b}_{2}$ of $i_{2}$, there is a best response strategy $\mathbf{b}_{\mathbf{1}}$ of $i_{1}$ such that the equilibrium allocation with $\left(\mathbf{b}_{\mathbf{1}}, \mathbf{b}_{\mathbf{2}}\right)$ is unique. Further, this best response strategy $\mathbf{b}_{\mathbf{1}}$ is given by

$$
b_{1 j}= \begin{cases}e_{1} & \text { if } j \in \arg \max \left\{b_{2 j}\right\} \\ 1-\frac{b_{2 j}}{b_{2}^{\max }} e_{2} & \text { otherwise }\end{cases}
$$

where the maximum utility of $i_{1}$ is $u_{1}\left(\mathbf{b}_{\mathbf{1}}, \mathbf{b}_{\mathbf{2}}\right)=\min _{j}\left\{\frac{1-\frac{b_{2 j}}{b_{2}^{\max } e_{2}}}{a_{1 j}}\right\}$.

For a market with $n$ buyers, we have the following result which follows from Theorem 1 directly.

Theorem 2. For any given market with $n$ buyers and reported bids $\mathbf{b}_{\mathbf{2}}, \ldots, \mathbf{b}_{\mathbf{n}}$ of $i_{2}, \ldots, i_{n}$, respectively, let $S=\left\{j \mid b_{i j}=b_{i}^{\max }, i \neq i_{1}\right\}$. If $S \neq \emptyset$, then a best response strategy $\mathbf{b}_{\mathbf{1}}$ of $i_{1}$ is given by

$$
b_{1 j}= \begin{cases}e_{1} & \text { if } j \in S \\ 1-\sum_{i \neq i_{1}} \frac{b_{i j}}{b_{i}^{\max }} e_{i} & \text { otherwise }\end{cases}
$$

where the maximum utility of $i_{1}$ is $u_{1}\left(\mathbf{b}_{\mathbf{1}}, \mathbf{b}_{\mathbf{2}}, \ldots, \mathbf{b}_{\mathbf{n}}\right)=\min _{j}\left\{\frac{1-\sum_{i \neq i_{1}} \frac{b_{i j}}{b_{i}^{\text {\#ax }} e_{i}}}{a_{1_{j}}}\right\}$, and the resulting equilibrium allocation is unique.

The following theorem is a sufficient condition for being a dominant strategy for all buyers to bid truthfully, i.e., $\mathbf{b}_{\mathbf{i}}=\mathbf{a}_{\mathbf{i}}$.

Theorem 3. For any fixed bid $\mathbf{b}_{-\mathbf{i}}$ of all the buyers except $i$, if there exists an item $j$ such that $a_{i j}=a_{i}^{\text {max }}$, and $b_{i^{\prime} j}=b_{i^{\prime}}^{\max }$ for all $i^{\prime} \neq i$, then bidding truthfully is dominant strategy for $i$.

The above theorem implies that if there is a common item on which all buyers have the largest weight, then that item "dominates" the utilities and it is impossible to increase one's allocation. 


\section{Incentive Ratio}

In this section, we will present the definition of incentive ratio and our main results.

Definition 1 (Incentive Ratio). For a given market $M$ and any fixed bids $\mathbf{b}_{-\mathbf{i}}$ of other buyers, let $u_{i}\left(\mathbf{a}_{\mathbf{i}}, \mathbf{b}_{-\mathbf{i}}\right)$ be the utility of $i$ when he bids truthfully, and $\max _{\mathbf{b}_{\mathbf{i}}} u_{i}\left(\mathbf{b}_{\mathbf{i}}, \mathbf{b}_{-\mathbf{i}}\right)$ be the largest possible utility of $i$ when he unilaterally changes his bid. ${ }^{6}$ Define

$$
R_{i}^{M}=\max _{\mathbf{b}_{-\mathbf{i}}} \frac{\max _{\mathbf{b}_{\mathbf{i}}} u_{i}\left(\mathbf{b}_{\mathbf{i}}, \mathbf{b}_{-\mathbf{i}}\right)}{u_{i}\left(\mathbf{a}_{\mathbf{i}}, \mathbf{b}_{-\mathbf{i}}\right)}
$$

to be the incentive ratio of $i$ in the market $M$.

Incentive ratio quantifies the benefit of strategic behaviors of each individual buyer. Let $R^{M} \triangleq \max _{i} R_{i}^{M}$ denote the largest individual incentive ratio of $M$. Our main result is the following.

Theorem 4 (main). For any given Leontief market $M$ and a buyer $i \in M$, his incentive ratio is smaller than 2, i.e., $R_{i}^{M}<2$. Thus, $R^{M}<2$.

The ratio given by the theorem is tight; the following example shows that $R^{M}$ can take on a value arbitrarily close to 2 .

Example 2 (Tight example). There are 2 buyers and 2 items with $\mathbf{a}_{\mathbf{1}}=(1-\epsilon, \epsilon)$ and $\mathbf{a}_{2}=\left(\frac{1}{2}-\epsilon, \frac{1}{2}+\epsilon\right)$. Their budgets are $e_{1}=4 \epsilon-4 \epsilon^{2}+\epsilon^{3}$ and $e_{2}=1-4 \epsilon+$ $4 \epsilon^{2}-\epsilon^{3}$, where $\epsilon>0$ is an arbitrarily small number. Assume $\mathbf{b}_{\mathbf{2}}=\mathbf{a}_{\mathbf{2}}$. When $i_{1}$ bids truthfully, his utility is $u_{1}=\frac{4 \epsilon-4 \epsilon^{2}+\epsilon^{3}}{1-\epsilon}$. If $i_{1}$ strategically bids

$$
\mathbf{b}_{\mathbf{1}}=\left(\frac{8 \epsilon-12 \epsilon^{2}+9 \epsilon^{3}-2 \epsilon^{4}}{1+2 \epsilon}, 4 \epsilon-4 \epsilon^{2}+\epsilon^{3}\right)
$$

then his utility will be

$$
u_{1}^{\prime}=\frac{8 \epsilon-12 \epsilon^{2}+9 \epsilon^{3}-2 \epsilon^{4}}{(1+2 \epsilon)(1-\epsilon)}
$$

and the incentive ratio is

$$
\frac{8-12 \epsilon+9 \epsilon^{2}-2 \epsilon^{3}}{4+4 \epsilon-7 \epsilon^{2}+2 \epsilon^{3}}
$$

which converges to 2 as $\epsilon$ approaches 0.

Before proving Theorem 4, we first establish a connection of the incentive ratios of different markets in terms of the number of buyers and items. These properties are of independent interests to reveal the effect of market sizes on strategic behaviors. We will prove the theorem at the end of the section.

\footnotetext{
${ }^{6}$ As mentioned earlier, equilibrium allocation may not be unique for different bids of $i$, which may lead to different true utilities for him. Our definition of incentive ratio is the strongest in the sense that it bounds the largest possible utility in all possible equilibrium allocations, which include, of course, a best possible allocation.
} 


\subsection{Incentive Ratio is Independent of the Number of Buyers}

In this subsection, we will show that the incentive ratio of Leontief markets is independent of the number of buyers.

Theorem 5. Incentive Ratio is independent of the number of buyers. That is, if $R_{n}=\max \left\{R^{M_{n}} \mid M_{n}\right.$ is a market with $n$ buyers $\}$, then $R_{n}=R_{n^{\prime}}$ for any $n>n^{\prime} \geq 2$. (Note that $R_{1}=1$.)

The proof is by showing that $R_{n}=R_{2}$ for any $n \geq 3$; it consists of two directions of reductions: one is from $R_{n}$ to $R_{2}$ and the other is from $R_{2}$ to $R_{n}$.

Reduction from $\boldsymbol{R}_{\boldsymbol{n}}$ to $\boldsymbol{R}_{\mathbf{2}}$. We construct a reduction from any market with $n$ buyers to a 2-buyer market, as the following lemma states. The reduction is by unifying $n-1$ buyers in the $n$-buyer market, and comparing the incentive ratio of the buyer whose utility functions are the same in both of the markets.

Lemma 2. For any n-buyer market $M_{n}$, there is a 2-buyer market $M_{2}$ such that $R^{M_{2}} \geq R^{M_{n}}$. This implies that $R_{2} \geq R_{n}$.

Reduction from $\boldsymbol{R}_{\mathbf{2}}$ to $\boldsymbol{R}_{\boldsymbol{n}}$. We can have a similar reduction from any market with 2 buyers to an $n$-buyer market, as the following lemma states. (Note that such a reduction is necessary for Leontief utility functions, since we cannot simply add dummy buyers and items as they may affect the allocations of existing items.)

Lemma 3. For any 2-buyer market $M_{2}$, there is an n-buyer market $M_{n}$ such that $R^{M_{n}} \geq R^{M_{2}}$. This implies that $R_{n} \geq R_{2}$.

\subsection{Incentive Ratio is Independent of the Number of items}

The following claim shows that the incentive ratio does not depend on the number of items as well.

Theorem 6. Incentive ratio is independent of the number of items.

\subsection{Proof of Theorem 4}

In this subsection, we will prove our main theorem. Given the properties of the incentive ratio established in the above subsections, it suffices to bound the incentive ratio for a 2-buyer 2-item market.

Let $M$ be a market with 2 buyers $i_{1}, i_{2}$ and 2 items $j_{1}, j_{2}$ with true utility vectors $\mathbf{a}_{\mathbf{1}}=\left(a_{11}, a_{12}\right)$ and $\mathbf{a}_{\mathbf{2}}=\left(a_{21}, a_{22}\right)$. We will only analyze the incentive ratio of $i_{1}$; the same argument applies to $i_{2}$. Suppose the reported utility vector of $i_{2}$ is $\mathbf{b}_{\mathbf{2}}=\left(b_{21}, b_{22}\right)$, and assume without loss of generality that $b_{21}<b_{22}$. According to Theorem 3, we can assume that $a_{11}>a_{12}$ (otherwise truthful strategy is dominant strategy for $i_{1}$ and his incentive ratio is 1 ). 
Let $\mathbf{b}_{\mathbf{1}}=\left(b_{11}, b_{12}\right)$ be $i_{1}$ 's best response for $i_{2}$ 's bid $\mathbf{b}_{\mathbf{2}}$. Let $u_{1}=u_{1}\left(\mathbf{a}_{\mathbf{1}}, \mathbf{b}_{\mathbf{2}}\right), u_{1}^{\prime}=$ $\left(\mathbf{b}_{\mathbf{1}}, \mathbf{b}_{\mathbf{2}}\right)$ and $u_{2}=u_{2}\left(\mathbf{a}_{\mathbf{1}}, \mathbf{b}_{\mathbf{2}}\right)$. Hence, the incentive ratio of $i_{1}$ is

$$
R_{1}^{M}=\frac{u_{1}^{\prime}}{u_{1}}=\frac{\min _{j}\left\{\frac{1-\frac{b_{2 j}}{b_{2}^{\max } e_{2}}}{a_{1 j}}\right\}}{u_{1}}=\frac{\min \left\{\frac{1-\frac{b_{21}}{b_{22}} e_{2}}{a_{11}}, \frac{e_{1}}{a_{12}}\right\}}{u_{1}}
$$

There are the following two cases.

$-\frac{b_{21}}{b_{22}}>e_{2}$, or equivalently, $e_{1}>1-\frac{b_{21}}{b_{22}}$. For this case, we will construct a new market $\tilde{M}$ where $a_{12}$ is replaced by $\tilde{a}_{12}=\min \left\{a_{12},\left(1-\frac{b_{22}}{b_{21}} e_{2}\right) \frac{a_{12}}{e_{1}}-\epsilon\right\}$, and all other parameters remain unchanged. Let $\tilde{u}_{1}=\tilde{u}_{1}\left(\tilde{\mathbf{a}}_{1}, \mathbf{b}_{2}\right)$, where $\tilde{\mathbf{a}}_{\mathbf{1}}=\left(a_{11}, \tilde{a}_{12}\right)$.

Recall that $\tilde{u}_{1} \leq \frac{e_{1}}{\tilde{a}_{1}^{\min }}=\frac{e_{1}}{\tilde{a}_{12}}, u_{2} \leq \frac{e_{2}}{b_{2}^{\min }}=\frac{e_{2}}{b_{21}}$, and by definition $\tilde{a}_{12}<$ $\left(1-\frac{b_{22}}{b_{21}} e_{2}\right) \frac{a_{12}}{e_{1}}$, we can verify that when $i_{1}$ bids truthfully in market $\tilde{M}$, for item $j_{2}$ we have

$$
\begin{aligned}
a_{12} u_{1}+b_{22} u_{2}-1 & =\tilde{a}_{12} u_{1}+b_{22} u_{2}-1 \\
& <\left(1-\frac{b_{22}}{b_{21}} e_{2}\right) \frac{a_{12}}{e_{1}} \cdot \frac{e_{1}}{a_{12}}+b_{22} \frac{e_{2}}{b_{21}}-1=0
\end{aligned}
$$

According to linear complementary conditions, $p_{2}=0$ and $p_{1}>0$. Recall that $p_{1}+p_{2}=e_{1}+e_{2}=1$; we get $p_{1}=1$. Hence, $\tilde{u}_{1}=\frac{e_{1}}{p_{1} a_{11}+p_{2} \tilde{a}_{12}}=$ $\frac{e_{1}}{a_{11}}, u_{2}=\frac{e_{2}}{b_{21}}$. Therefore, $\tilde{u}_{1}=\frac{e_{1}}{a_{11}}=\frac{e_{1}}{a_{1}^{\max }} \leq u_{1}$. Hence,

$$
R_{1}^{M}=\frac{\min _{j}\left\{\frac{1-\frac{b_{2 j}}{b_{2}} e_{2}}{a_{1 j}}\right\}}{u_{1}} \leq \frac{\min \left\{\frac{1-\frac{b_{21}}{b_{22}} e_{2}}{a_{11}}, \frac{e_{1}}{a_{12}}\right\}}{\tilde{u}_{1}} \leq \frac{\min \left\{\frac{1-\frac{b_{21}}{b_{22}} e_{2}}{a_{11}}, \frac{e_{1}}{\tilde{a}_{12}}\right\}}{\frac{e_{1}}{a_{11}}} \leq R_{1}^{\tilde{M}}
$$

Now we can get the ratio of $\tilde{M}$. For item $j_{2}$, we have

$$
a_{1 j} u_{1}+b_{2 j} u_{2}-1=\tilde{a}_{12} u_{1}+b_{22} u_{2}-1=\tilde{a}_{12} \frac{e_{1}}{a_{11}}+b_{22} \frac{e_{2}}{b_{21}}-1<0
$$

Thus, $e_{1}>\frac{a_{11}\left(b_{22}-b_{21}\right)}{a_{11} b_{22}-\tilde{a}_{12} b_{21}}$. Recall that we have $\frac{b_{21}}{b_{22}}>e_{2}$, which is equivalent to $e_{1}>1-\frac{b_{21}}{b_{22}}$. In addition, $\frac{a_{11}\left(b_{22}-b_{21}\right)}{a_{11} b_{22}-\tilde{a}_{12} a b_{21}}>1-\frac{b_{21}}{b_{22}}$, we conclude the constraint for $e_{1}$ satisfies

$$
e_{1}>\frac{a_{11}\left(b_{22}-b_{21}\right)}{a_{11} b_{22}-\tilde{a}_{12} b_{21}}
$$

Now comparing the two terms of buyer $i_{1}$ 's utility when he uses best response strategy, we have

$$
\begin{aligned}
\frac{1-\frac{b_{21}}{b_{22}} e_{2}}{a_{11}}-\frac{e_{1}}{\tilde{a}_{12}} & =\frac{1}{a_{11}}\left[\left(1-\frac{b_{21}}{b_{22}}\right)-\frac{a_{11} b_{22}-\tilde{a}_{12} b_{21}}{\tilde{a}_{12} b_{22}} e_{1}\right] \\
& <\frac{1}{a_{11}}\left[\left(1-\frac{b_{21}}{b_{22}}\right)-\frac{a_{11} b_{22}-\tilde{a}_{12} b_{21}}{\tilde{a}_{12} b_{22}} \cdot \frac{a_{11}\left(b_{22}-b_{21}\right)}{a_{11} b_{22}-\tilde{a}_{12} b_{21}}\right] \\
& =\frac{1}{a_{11}}\left(1-\frac{b_{21}}{b_{22}}\right)\left(1-\frac{a_{11}}{\tilde{a}_{12}}\right)<0
\end{aligned}
$$


Therefore,

$$
\begin{aligned}
R & =\frac{u_{1}^{\prime}}{u_{1}}=\frac{\min _{j}\left\{\frac{1-\frac{b_{2 j}}{b_{2}^{\max } e_{2}}}{a_{1 j}}\right\}}{u_{1}} \leq \frac{\frac{1-\frac{b_{21}}{b_{22}} e_{2}}{a_{11}}}{\frac{e_{1}}{a_{11}}}=\frac{1-\frac{b_{21}}{b_{22}} e_{2}}{e_{1}}=\frac{b_{22}-b_{21}}{b_{22}} \frac{1}{e_{1}}+\frac{b_{21}}{b_{22}} \\
& <\frac{b_{22}-b_{21}}{b_{22}} \cdot \frac{a_{11} b_{22}-\tilde{a}_{12} b_{21}}{a_{11}\left(b_{22}-b_{21}\right)}+\frac{b_{21}}{b_{22}}=1+\frac{b_{21}}{b_{22}}\left(1-\frac{\tilde{a}_{12}}{a_{11}}\right)<1+1=2
\end{aligned}
$$

where the last inequality is due to $0<b_{22}-b_{21}<\epsilon$ and $0<\tilde{a}_{12}<\epsilon$, as $\epsilon$ approaches 0 .

- $\frac{b_{21}}{b_{22}} \leq e_{2}$, or equivalently, $e_{1} \leq 1-\frac{b_{21}}{b_{22}}$. Since $\frac{b_{21}}{b_{22}} \leq e_{2}$, we know that when buyer $i_{1}$ bids truthfully, $u_{2}<\frac{e_{2}}{b_{21}}$. Otherwise if $u_{2}=\frac{e_{2}}{b_{21}}$, then for item $j_{2}$, $a_{12} u_{1}+b_{22} \frac{e_{2}}{b_{21}}-1 \geq a_{12} u_{1}+1-1>0$, which is a contradiction. On the other hand, if $u_{2}=\frac{e_{2}}{b_{22}}$, then buyer $i_{1}$ cannot improve his utility any further and his incentive ratio is 1 . Therefore, the utilities satisfy

$$
\frac{e_{1}}{a_{11}}<u_{1}<\frac{e_{1}}{a_{12}}, \quad \frac{e_{2}}{b_{22}}<u_{2}<\frac{e_{2}}{b_{21}}
$$

According to the KKT conditions, we have

$$
\begin{gathered}
0<p_{1}<1, \quad 0<p_{2}<1 \\
\left\{\begin{array}{l}
a_{11} u_{1}+b_{21} u_{2}-1=0 \\
a_{12} u_{1}+b_{22} u_{2}-1=0
\end{array}\right.
\end{gathered}
$$

Thus,

$$
u_{1}=\frac{b_{22}-b_{21}}{a_{11} b_{22}-a_{12} b_{21}}, \quad u_{2}=\frac{a_{11}-a_{12}}{a_{11} b_{22}-a_{12} b_{21}}
$$

Plug it into (3), we have

$$
\frac{a_{12}\left(b_{22}-b_{21}\right)}{a_{11} b_{22}-a_{12} b_{21}}<e_{1}<\frac{a_{11}\left(b_{22}-b_{21}\right)}{a_{11} b_{22}-a_{12} b_{21}}
$$

Since $1-\frac{b_{21}}{b_{22}}<\frac{a_{11}\left(b_{22}-b_{21}\right)}{a_{11} b_{22}-a_{12} b_{21}}$, we conclude the constraint for $e_{1}$ is,

$$
\frac{a_{12}\left(b_{22}-b_{21}\right)}{a_{11} b_{22}-a_{12} b_{21}}<e_{1} \leq 1-\frac{b_{21}}{b_{22}}
$$

Thus,

$$
\begin{aligned}
\frac{1-\frac{b_{21}}{b_{22}} e_{2}}{a_{11}}-\frac{e_{1}}{a_{12}} & =\frac{1}{a_{11}}\left[\left(1-\frac{b_{21}}{b_{22}}\right)-\frac{a_{11} b_{22}-a_{12} b_{21}}{a_{12} b_{22}} e_{1}\right] \\
& <\frac{1}{a_{11}}\left[\left(1-\frac{b_{21}}{b_{22}}\right)-\frac{a_{11} b_{22}-a_{12} b_{21}}{a_{12} b_{22}} \cdot \frac{a_{12}\left(b_{22}-b_{21}\right)}{a_{11} b_{22}-a_{12} b_{21}}\right] \\
& =\frac{1}{a_{11}}\left[\left(1-\frac{b_{21}}{b_{22}}\right)-\left(1-\frac{b_{21}}{b_{22}}\right)\right]=0
\end{aligned}
$$


Therefore,

$$
\begin{aligned}
R_{1}^{M} & =\frac{u_{1}^{\prime}}{u_{1}}=\frac{\min _{j}\left\{\frac{1-\frac{b_{2 j}}{b_{2}^{\text {max }} e_{2}}}{a_{1 j}}\right\}}{u_{1}}=\frac{\frac{1-\frac{b_{21}}{b_{22}} e_{2}}{a_{11}}}{\frac{b_{22}-b_{21}}{a_{11} b_{22}-a_{12} b_{21}}} \\
& =\frac{a_{11} b_{22}-a_{12} b_{21}}{a_{11}\left(b_{22}-b_{21}\right)}\left(1-\frac{b_{21}}{b_{22}}+\frac{b_{21}}{b_{22}} e_{1}\right) \\
& \leq \frac{a_{11} b_{22}-a_{12} b_{21}}{a_{11}\left(b_{22}-b_{21}\right)}\left(\frac{b_{22}-b_{21}}{b_{22}}+\frac{b_{21}}{b_{22}} \cdot \frac{b_{22}-b_{21}}{b_{22}}\right) \\
& =\left(1-\frac{a_{12}}{a_{11}} \cdot \frac{b_{21}}{b_{22}}\right)\left(1+\frac{b_{21}}{b_{22}}\right)<(1-0)(1+1)=2
\end{aligned}
$$

where the last inequality is due to $0<b_{22}-b_{21}<\epsilon$ and $0<a_{12}<\epsilon$, as $\epsilon$ approaches 0 .

This completes the proof.

\section{Conclusions}

We introduce the concept of incentive ratio to characterize the extent to which utilities can be increased by strategic behaviors of individuals in a marketplace. It would be interesting to study the incentive ratio for other market models, e.g., linear, Cobb-Douglas, or general, CES utility functions, as well as Arrow-Debreu markets. The definition of incentive ratio can be generalized to other mechanism design settings. For example, if a mechanism is incentive compatible, its incentive ratio is one. A notion similar to incentive ratio is approximate truthfulness, which has been considered in, e.g., [22, 20,3,18]. The concept of incentive ratio focuses on individual participant rather than the worst case analysis of the whole market. In particular, for markets with asymmetric information, the incentive ratios of different individuals could be very different, depending on their knowledge and unbalanced situation in the market. The incentive ratio defined in our paper characterizes robustness of incentives for individuals, and has potential applications in other settings.

\section{References}

1. B. Adsul, S. Babu, J. Garg, R. Mehta, M. Sohoni, Nash Equilibria in Fisher Market, SAGT 2010, 30-41.

2. E. Anshelevich, A. Dasgupta, J. M. Kleinberg, Éva Tardos, T. Wexler, T. Roughgarden, The Price of Stability for Network Design with Fair Cost Allocation, SIAM Journal on Computing, V.38(4): 1602-1623, 2008.

3. A. Archer, C. Papadimitriou, K. Talwar, E. Tardos, An Approximate Truthful Mechanism for Combinatorial Auctions with Single Parameter Agents, SODA 2003, 205-214. 
4. K. J. Arrow, H. Chenery, B. Minhas, R. Solow, Capital-Labor Substitution and Economic Efficiency, The Review of Economics and Statistics, V.43(3), 225-250, 1961.

5. K. Arrow, G. Debreu, Existence of an Equilibrium for a Competitive Economy, Econometrica, V.22, 265-290, 1954.

6. M. S. Bazaraa, H. D. Sherali, C. M. Shetty, Nonlinear Programming: Theory and Algorithms, John Wiley \& Sons, 2006.

7. W. Brainard, H. E. Scarf, How to Compute Equilibrium Prices in 1891, Cowles Foundation Discussion Paper, 2000.

8. N. Chen, X. Deng, X. Sun, A. C. Yao, Fisher Equilibrium Price with Concave Utility Functions, ESA 2004, 169-179.

9. B. Codenotti, K. R. Varadarajan, Efficient Computation of Equilibrium Prices for Markets with Leontief Utilities, ICALP 2004, 371-382.

10. B. Codenotti, A. Saberi, K. Varadarajan, Y. Ye, Leontief Economies Encode Nonzero Sum Two-Player Games, SODA 2006, 659-667.

11. X. Deng, C. Papadimitriou, S. Safra, On the Complexity of Equilibria, STOC 2002, 67-71.

12. N. Devanur, R. Kannan, Market Equilibria in Polynomial Time for Fixed Number of Goods or Agents, FOCS 2008, 45-53.

13. N. Devanur, C. Papadimitriou, A. Saberi, V. Vazirani, Market Equilibrium via a Primal-Dual Algorithm for a Convex Program, JACM, V.55(5), 2008.

14. B. C. Eaves, Finite Solution of Pure Trade Markets with Cobb-Douglas Utilities, Mathematical Programming Study, V.23, 226-239, 1985.

15. E. Eisenberg, D. Gale, Consensus of Subjective Probabilities: The Pari-Mutuel Method, Annals Of Mathematical Statistics, V.30, 165-168, 1959.

16. D. Garg, K. Jain, K. Talwar, V. Vazirani, A Primal-Dual Algorithm for Computing Fisher Equilibrium in the Absence of Gross Substitutability Property, Theoretical Computer Science, V.378, 143-152, 2007.

17. K. Jain, A polynomial Time Algorithm for Computing an Arrow-Debreu Market Equilibrium for Linear Utilities, SIAM Journal on Computing, V.37(1), 303-318, 2007.

18. A. Kothari, D. Parkes, S. Suri, Approximately-Strategyproof and Tractable Multiunit Auctions, Decision Support Systems, V.39(1), 105-121, 2005.

19. E. Koutsoupias, C. H. Papadimitriou, Worst-Case Equilibria, STACS 1999, 404413.

20. D. Lehmann, L. O'Callaghan, Y. Shoham, Truth Revelation in Approximately Efficient Combinatorial Auctions, JACM, V.49(5), 577602, 2002.

21. D. Roberts, A. Postlewaite, The Incentives for Price-Taking Behavior in Large Exchange Economies, Econometrica, V.44, 113-127, 1976.

22. J. Schummer, Almost Dominant Strategy Implementation, MEDS Department, Northwestern University, Discussion Papers 1278, 1999.

23. R. Solov, A Contribution to the Theory of Economic Growth, Quarterly Journal of Economics, V.70, 65-94, 1956.

24. H. Varian, Microeconomic Analysis, W. W. Norton \& Company, 1992.

25. Y. Ye, A Path to the Arrow-Debreu Competitive Market Equilibrium, Mathematical Programming, V.111(1-2), 315-348, 2008. 